\title{
Multimorbidity and Fatigue in Rheumatoid Arthritis: A Cross-Sectional Study of a Population-Based Cohort
}

John M. Davis III (D) · Elena Myasoedova · Tina M. Gunderson •

Cynthia S. Crowson

Received: August 13, 2020 / Accepted: October 14, 2020 / Published online: October 28, 2020

(C) The Author(s) 2020

\section{ABSTRACT}

Introduction: The objective was to evaluate the relationships between multimorbidity and overall fatigue as well as fatigue subdomains in patients with rheumatoid arthritis (RA).

Methods: A cross-sectional study of a population-based cohort of patients with RA was performed. Fatigue was assessed using the Bristol Rheumatoid Arthritis Fatigue Multidimensional Questionnaire (BRAF-MDQ). Patients' medical records were reviewed for 25 chronic comorbidities prior to the BRAF-MDQ. Linear and logistic regression models were used to estimate the differences in BRAF-MDQ total and subdomain (physical, living, cognitive, and emotional) scores associated with multimorbidity, adjusting for age, sex, disease duration, obesity, smoking, C-reactive protein, and RA autoantibodies. Higher BRAF-MDQ scores indicate greater fatigue severity.

Electronic Supplementary Material The online version of this article (https://doi.org/10.1007/s40744020-00247-y) contains supplementary material, which is available to authorized users.

J. M. Davis III $(\bowtie) \cdot$ E. Myasoedova $\cdot$ C. S. Crowson Division of Rheumatology, Mayo Clinic, Rochester, MN, USA

e-mail: davis.john4@mayo.edu

T. M. Gunderson · C. S. Crowson

Department of Health Sciences Research, Mayo

Clinic, Rochester, MN, USA
Results: The cohort included 192 patients, median age 62 years, and median RA duration 13 years. Multimorbidity was common with 93 (48\%) having $\geq 2$ comorbidities, and 27 (14\%) having $\geq 4$ comorbidities. The median BRAFMDQ total score was 9 (interquartile range 3-18), with higher scores indicating greater fatigue. Patients with $\geq 4$ comorbidities had higher total BRAF-MDQ scores (median 16.5, interquartile range: 6.8-24.8) than patients with $<4 \quad$ comorbidities $\quad(7.5, \quad 2.8-16.0$; $p=0.014)$. Each additional comorbidity was associated with a 2.33 (95\% confidence interval [CI] 1.10-3.56) unit increase in total BRAF-MDQ score $(p<0.001)$, and the presence of $\geq 4$ comorbidities was associated with a $9.33(95 \%$ CI 3.92-14.7) unit increase in total BRAF-MDQ score. Multimorbidity was significantly associated with all four fatigue subdomains in adjusted models.

Conclusions: Multimorbidity is associated with increased fatigue in patients with RA. The findings suggest that interventions targeting multimorbidity could help alleviate treatmentrefractory fatigue in patients with RA and other rheumatic diseases.

Keywords: Autoimmune disease; Comorbidity; Fatigue subdomains; Health care burden; Multimorbidity; Rheumatic disease 


\section{Key Summary Points}

Why carry out this study?

Previous studies have reported that multimorbidity is associated with the severity of fatigue as defined by visual or numeric scales.

There is an unmet need to more deeply understand effects of multimorbidity on various facets of the fatigue experience.

\section{What was learned from this study?}

In this study, we have shown that the presence of substantial multimorbidity, defined as the presence of $\geq 4$ comorbidities, is associated with severe fatigue after adjusting for important confounders.

Multimorbidity is an important contributor to the experience of fatigue among patients with RA, including the physical, living, cognitive, and emotional aspects of the disease.

\section{DIGITAL FEATURES}

This article is published with digital features to facilitate understanding of the article. To view the digital features for this article go to https:// doi.org/10.6084/m9.figshare.13083962.

\section{INTRODUCTION}

Despite treatment advances, fatigue continues to have a major impact on people living with rheumatoid arthritis (RA) [1]. The prevalence of clinically important fatigue (e.g., $>40 \mathrm{~mm}$ on a $100-\mathrm{mm}$ visual analog scale or SF-36 vitality score $<35)$ is around $40-50 \%[2,3]$. Persistent fatigue occurs in more than $50 \%$ of patients after 10 years of follow-up and is more common among women than men (60 vs. 40\%) [4, 5]. Key mediators of RA fatigue are inflammation, pain, mental health and coping styles, physical activity, sleep quality, deconditioning and muscle atrophy, metabolic changes, and functional limitations and disability [6-8]. Importantly, fatigue is a pervasive, unpredictable, and often inexplicable problem for patients with RA that greatly interferes with their functional status and quality of life $[9,10]$.

Advances in treat-to-target strategies and targeted therapies have so far not ameliorated this problem as many RA patients report persistent fatigue despite attainment of clinical remission on biologic therapies $[11,12]$. Investigation of longitudinal data from the Early RA Network (ERAN) and Early RA Study (ERAS) in the United Kingdom for secular trends in disease trajectories for patients diagnosed in 1990, 2002, or 2010 have shown that while the initial 5 -year course for inflammatory disease activity has improved over the decades, improvements in fatigue in early RA have been much smaller in magnitude and not statistically significant $[13,14]$. In a recent large administrative claims study, patients with diagnostic codes for RA and fatigue had higher all-cause and RA-specific health care utilization and costs compared to matched controls without fatigue, underscoring not only the burden of fatigue for patients but also for health care systems [15].

Multimorbidity, the presence of two or more chronic diseases, is increasingly recognized as an important issue in RA that affects treatment and outcomes $[16,17]$. In an analysis of the aforementioned ERAN and ERAS studies, the lack of improvement over the years in fatigue for patients with early RA was observed in parallel with a dramatic rise in comorbidity burden (i.e., $\geq 1$ ) from $29 \%$ in 1990 to $50.7 \%$ in 2010 , suggesting that multimorbidity may be an important contributor to fatigue [18]. In the large COMORA (COMOrbidity in Rheumatoid Arthritis) study, involving 3920 patients in 17 countries, the top comorbidities were depression $(15 \%)$, asthma $(6.6 \%)$, cardiovascular disease $(6 \%)$, malignancies $(4.5 \%)$, and chronic obstructive pulmonary disease (3.5\%) [19]. The prevalence of multimorbidity is as high as twothirds of patients with RA in some studies [17]. The numeric count of comorbidities ('multimorbidity index') is associated with a stepwise 
reduction in physical function as assessed by the Health Assessment Questionnaire (HAQ) and health-related quality of life as assessed using the SF-36 or EQ-5D instruments [20, 21]. Multimorbidity has been associated with reduced probability of clinical remission, accounting for RA disease characteristics and disease-modifying therapies [22].

However, relatively little is known about the relationship between multimorbidity and RA fatigue. Recognizing that fatigue is a complex and multidimensional experience, there is an unmet need to delve deeper into the effects of multimorbidity on the various facets of the fatigue experience for patients. Therefore, the objective of this study was to determine the associations between multimorbidity and overall fatigue as well as known fatigue subdomains in patients with RA.

\section{METHODS}

\section{Study Design and Participants}

A prospective, cross-sectional study of a community population-based cohort of adult residents $\geq 18$ years of age in Olmsted County, Minnesota, with RA was performed. The cohort was previously assembled using the resources of the Rochester Epidemiology Project, a population-based medical records linkage system of the complete inpatient and outpatient medical records from all healthcare providers in Olmsted County [23]. Participants fulfilled the 1987 American College of Rheumatology (ACR) classification criteria for RA [24]. Both incident and prevalent cases of RA living in Olmsted County in 2012-2014 were invited to participate in this prospective study, which involved a single study visit where a survey was completed. From the total population of patients alive and eligible, 192 of 296 agreed to participate in this study. The Mayo Clinic (IRB number 06-005445) and Olmsted Medical Center (IRB number 039-omc-06) institutional review boards approved this research study. The study was conducted in accordance with the Helsinki Declaration of 1964 and its later amendments. All participants provided written informed consent. No protected health information of research participants is disclosed in this manuscript.

\section{Data Collection}

A review of medical records was performed by trained nurse abstractors. Data collection included participant demographics, RA duration, body mass index (BMI, $\mathrm{kg} / \mathrm{m}^{2}$ ), smoking status (ever/never), serological status for rheumatoid factor (RF) and anti-cyclic citrullinated peptide (anti-CCP) antibodies, C-reactive protein (CRP, $\mathrm{mg} / \mathrm{l})$, and current use of conventional synthetic disease-modifying antirheumatic drugs (DMARDs), biologic agents, or prednisone. During a research visit, patients completed the Routine Assessment of Patient Index Data 3 (RAPID-3) to assess disease severity; according to the RAPID-3 scores, disease severity was classified as remission (0.0-3.0), low (3.1-6.0), moderate (6.1-12.0), or high (12.1-30.0) disease severity [25].

\section{Measurement of Fatigue}

The Bristol Rheumatoid Arthritis Fatigue Multidimensional Questionnaire (BRAF-MDQ) was used to comprehensively assess fatigue and related health domains [26-30]. This 20-item questionnaire encompasses a total score (range, 0-70) as well as physical (range, 0-22), living with fatigue (range, 0-21), cognitive (range, 0-15), and emotional (range, 0-12) subdomain scores. Higher scores reflect greater fatigue severity. A salient attribute of this scale is that it was developed in collaboration with patient research partners [31]. The BRAF-MDQ has been shown to be valid, reliable, and sensitive to change for use in RA clinical research and practice $[27,28]$.

\section{Definition of Multimorbidity}

Diagnostic codes from all healthcare providers in Olmsted County for a period of 5 years prior to the survey date were used to define 25 chronic medical conditions based on a combination of the Charlson Comorbidity Index [32], 
Elixhauser Comorbidity Index [33], and Rheumatic Disease Comorbidity Index [34]. At least two diagnostic codes at least 30 days apart were required to define each comorbidity. This list included cancer, cerebrovascular disease, chronic pulmonary disease, myocardial infarction, congestive heart failure, valvular disease, dementia, diabetes mellitus, liver disease, paralysis, peripheral vascular disorders, renal failure, alcohol and substance abuse, coagulopathy, deficiency anemias, depression, hypertension, hypothyroidism, psychoses, other neurological disorders, pulmonary circulation disorders, and spine, hip or leg fractures. Human immunodeficiency virus (HIV)/acquired immunodeficiency syndrome (AIDS) and metastatic cancer were also included, but were not observed. Rheumatic diseases (i.e., lupus) and RA extraarticular manifestations (e.g., interstitial lung disease, secondary Sjögren's syndrome, vasculitis) were not included in the definition of multimorbidity. Multimorbidity was assessed using the count of comorbidities other than RA. Substantial multimorbidity was defined as $\geq 4$ comorbidities.

\section{Statistical Analysis}

Descriptive statistics (counts/percentages, medians/interquartile ranges [IQR], etc.) were used to summarize patient characteristics. Comparisons between different multimorbidity groups (i.e., $<4$ comorbidities vs. $\geq 4$ ) were performed using Chi-square, Fisher's exact, and Mann-Whitney-Wilcoxon tests. Linear and logistic regression models were used to estimate the association of fatigue with multimorbidity, adjusting for age, sex, duration of RA, obesity $\left(\mathrm{BMI} \geq 30 \mathrm{~kg} / \mathrm{m}^{2}\right)$, smoking status, CRP, and $\mathrm{RF} /$ anti-CCP positivity. This list of adjustors was defined a priori based on known or potential associations either with RA disease activity or severity, fatigue, or multimorbidity. Linear models were fit for the total BRAF-MDQ score and each subdomain score. CRP values were transformed using the natural logarithm (i.e., $\ln [\mathrm{CRP}])$ because the CRP has a highly rightskewed distribution; the transformed values provide better adjustment in the model. For the logistic model, BRAF-MDQ scores were dichotomized into the upper quartile and lower three quartiles. Two sensitivity analyses were performed: one excluded prevalent RA cases in order to determine if the associations between multimorbidity and fatigue were different for incident cases, and another adjusted for RA medications to minimize potential confounding effects on the associations between multimorbidity and fatigue. Analyses were performed using SAS version 9.4 (SAS Institute, Cary, NC, USA) and R 3.6.1 (R Foundation for Statistical Computing, Vienna, Austria).

\section{RESULTS}

The study included a total of 192 patients with RA from a population-based cohort in Olmsted County, Minnesota. Table 1 shows demographics and clinical characteristics, according to the absence $(<4)$ or presence of substantial multimorbidity ( $\geq 4$ comorbidities). Patients with substantial multimorbidity were numerically older (mean 66.4 vs. 62.5 years), but the age difference was not statistically significant $(p=0.078)$. There were no differences between the groups without or with substantial multimorbidity for BMI, smoking status, or RF/antiCCP positivity. The patients with substantial multimorbidity had higher disease severity and functional disability, according to both the RAPID-3 (median 11.0, IQR 9.0-14.3 vs. median 5.1, IQR 1.8-10.1, $p<0.001)$ and HAQ disability index (median 1.1, IQR 0.7-1.8) vs. median 0.4 IQR $0.0-1.0, p<0.001)$, compared to patients without substantial multimorbidity. There were no statistically significant or clinically meaningful differences in current usage of conventional synthetic DMARDs, biologics, or prednisone between the groups.

Multimorbidity was found to be associated with greater fatigue as assessed by the BRAFMDQ total score and subdomains (Table 2, Fig. 1). The median (IQR) for the BRAF-MDQ total score was significantly higher among patients with substantial multimorbidity at 16.5 (6.8-24.8) compared to patients with $<4$ comorbidities at $7.5(2.8-16.0 ; p=0.014)$. The proportions of patients in the highest quartile 
Table 1 Demographics and clinical characteristics of 192 patients with RA, according to the absence $(<4)$ or presence of substantial multimorbidity ( $\geq 4$ comorbidities)

\begin{tabular}{lllr}
\hline Variable & $<\mathbf{4}(\boldsymbol{N}=\mathbf{1 7 5})$ & $\mathbf{2} \mathbf{4}(\boldsymbol{N}=\mathbf{2 7})$ & $\boldsymbol{p}$ value \\
\hline Age, years & $61.9(54.6,70.9)$ & $65.6(59.9,74.1)$ & 0.078 \\
Female sex & $125(76 \%)$ & $19(70 \%)$ & 0.549 \\
RA duration, years & $12.7(8.1,18.9)$ & $11.9(6.3,23.4)$ & 0.718 \\
BMI, kg/m ${ }^{2}$ & $29.2(24.3,33.3)$ & $30.3(26.0,35.4)$ & 0.265 \\
Obesity $\left(\mathrm{BMI} \geq 30 \mathrm{~kg} / \mathrm{m}^{2}\right)$ & $73(45 \%)$ & $14(52 \%)$ & 0.516 \\
Smoking, ever & $9(5 \%)$ & $3(11 \%)$ & 0.260 \\
RF or anti-CCP, pos & $115(70 \%)$ & $16(59 \%)$ & 0.280 \\
C-reactive protein, mg/l & $2.1(0.9,4.5)$ & $3.0(0.9,7.1)$ & 0.196 \\
HAQ & $0.4(0.0,1.0)$ & $1.1(0.7,1.8)$ & $<0.001$ \\
RAPID-3 & $5.1(1.8,10.1)$ & $11.0(9.0,14.3)$ & $<0.001$ \\
DMARDs, current use & & & 0.322 \\
Methotrexate & $87(53 \%)$ & $17(63 \%)$ & 0.421 \\
Other DMARDs & $60(36 \%)$ & $12(44 \%)$ & 0.781 \\
TNF biologics & $28(17 \%)$ & $4(15 \%)$ & $3(11 \%)$ \\
Non-TNF biologics & $6(4 \%)$ & $10(37 \%)$ & 0.088 \\
Prednisone, use & $36(22 \%)$ & $0.0(0.0,4.5)$ & 0.086 \\
Prednisone, mg dose & $0.0(0.0,0.0)$ & & 0.065 \\
\hline
\end{tabular}

Values are median (Q1, Q3) or number (\%)

$R A$ rheumatoid arthritis, $B M I$ body mass index, $R F$ rheumatoid factor, $C C P$ cyclic citrullinated peptide, $H A Q$, Health Assessment Questionnaire, RAPID-3 Routine Assessment of Patient Index Data 3, DMARDs disease-modifying antirheumatic drugs, $T N F$ tumor necrosis factor

${ }^{\text {a }}$ Number (\%) in the top quartile for each BRAF-MDQ domain

of the BRAF-MDQ total score and subscores were determined. Twelve of 27 (46\%) patients with substantial multimorbidity were in the highest quartile of the BRAF-MDQ total score as compared to 36 of 175 (22\%) of the patients with $<4$ comorbidities $(p=0.008)$. According to the BRAF-MDQ, the group with substantial multimorbidity had higher scores for the physical, living, cognition, and emotion domains of fatigue than the patients with $<4$ comorbidities. The most common comorbidities overall were hypertension $(N=89,46 \%)$, hypothyroidism $(N=46,24 \%)$, and deficiency anemias $(N=33,17 \%)$. A similar hierarchy of the most common comorbidities was observed in each group, i.e., in patients with and without multimorbidity. However, as anticipated, each of these were more common in the group with substantial multimorbidity $(85,37$, and $52 \%$, respectively) than in the group with $<4$ comorbidities $(40,22$, and $12 \%$, respectively). Additionally, diabetes mellitus, peripheral vascular disorders, and psychoses showed similar prevalence to hypothyroidism in the substantial multimorbidity group (i.e., $37 \%$ for each), but were substantially lower in the group without multimorbidity $(6,2$, and $10 \%$, respectively).

Multimorbidity remained associated with increased overall fatigue and its subdomains after adjustment for potential confounders in 
Table 2 Total and subdomain scores on the Bristol Rheumatoid Arthritis Fatigue Multidimensional Questionnaire for 192 patients with RA, according to the absence $(<4)$ or presence of substantial multimorbidity ( $\geq 4$ comorbidities)

\begin{tabular}{lllr}
\hline Variable $^{\mathbf{a}}$ & $<\mathbf{4}$ comorbidities $(\boldsymbol{N}=\mathbf{1 7 5})$ & $\mathbf{2}$ c comorbidities $(\boldsymbol{N}=\mathbf{2 7})$ & $\boldsymbol{p}$ value \\
\hline Total score & $7.5(2.8,16.0)$ & $16.5(6.8,24.8)$ & 0.014 \\
Total & $36(22 \%)$ & $12(46 \%)$ & 0.008 \\
Physical & $47(28 \%)$ & $14(52 \%)$ & 0.016 \\
Living & $47(29 \%)$ & $13(50 \%)$ & 0.030 \\
Cognition & $50(30 \%)$ & $13(48 \%)$ & 0.067 \\
Emotion & $49(30 \%)$ & $17(63 \%)$ & $<0.001$ \\
\hline
\end{tabular}

$R A$ rheumatoid arthritis

${ }^{a}$ Numbers in table are median (interquartile range) or number (\%) in the highest quartile for each BRAF-MDQ domain

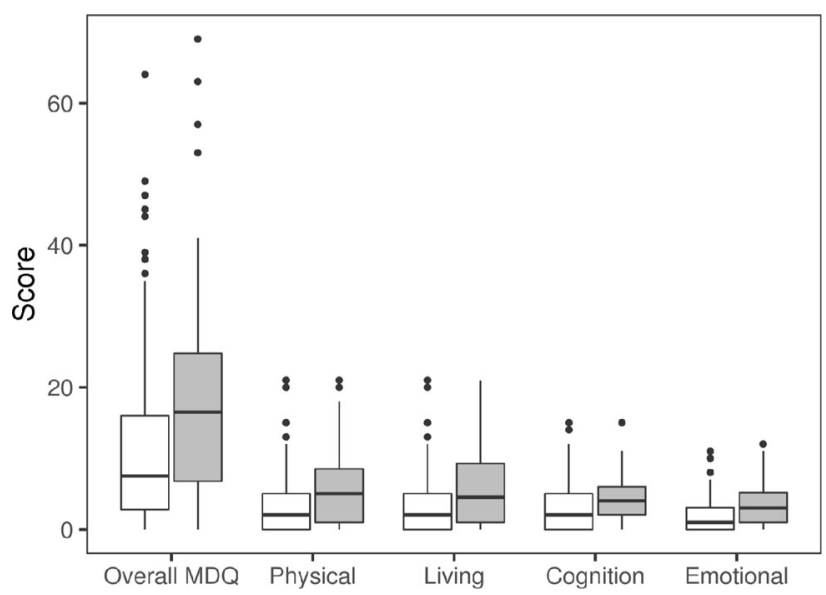

Fig. 1 Boxplot of the BRAF-MDQ total score and domain subscores, according to the presence of multimorbidity (white boxes show 0-3 comorbidities and grey boxes

regression models, with the important exception of the RAPID-3 (Table 3). The presence of substantial multimorbidity was significantly associated with overall BRAF-MDQ fatigue, adjusting for age, sex, RA disease duration, obesity, smoking, RF/anti-CCP, and CRP (Table 3, Model 1). The adjusted odds ratio (OR) and 95\% confidence interval (CI) for the association between substantial multimorbidity and the highest quartile of the BRAF-MDQ total score was 3.18 (95\% CI 1.26-8.04) (Table 3, Model 1). Similarly, both the number of comorbidities and substantial multimorbidity were associated with increased fatigue for each show $4+$ comorbidities). Box limits represent the interquartile range (IQR 25th-75th percentile) with whisker limits set at $1.5 \times \mathrm{IQR}$

of the BRAF-MDQ subdomains, including the physical, living, cognition, and emotion domains, adjusting for potential confounders (Table 4). However, after additional adjustment of the model for RAPID-3 (Table 3, Model 2), the association between substantial multimorbidity and the overall BRAF-MDQ became not statistically significant (adjusted OR 1.76; 95\% CI 0.57-5.27). In this model, age became a statistically significant determinant of fatigue (adjusted OR 0.59; 95\% CI 0.40-0.86), meaning greater age was associated with lower levels of overall BRAF-MDQ fatigue. 
Table 3 Adjusted association between multimorbidity and BRAF-MDQ total score

\begin{tabular}{llr}
\hline Variable & OR (95\% CI) & Model 2 \\
\cline { 2 - 3 }$\geq 4$ comorbidities & Model 1 & $1.76(0.57,5.27)$ \\
Smoking, ever & $3.18(1.26,8.04)$ & $0.70(0.10,4.41)$ \\
Ln(CRP), mg/l & $1.83(0.39,8.04)$ & $1.21(0.81,1.81)$ \\
Obesity & $1.26(0.92,1.74)$ & $0.72(0.30,1.68)$ \\
RA duration (per 10 years) & $1.08(0.52,2.23)$ & $0.85(0.53,1.32)$ \\
Age (per 10 years) & $0.92(0.59,1.38)$ & $0.59(0.40,0.86)$ \\
RF or anti-CCP positive & $0.76(0.55,1.03)$ & $0.73(0.30,1.79)$ \\
Male sex & $0.71(0.34,1.54)$ & $0.90(0.81,1.81)$ \\
RAPID-3 & $0.69(0.27,1.60)$ & $1.24(1.16,1.35)$ \\
\hline
\end{tabular}

Multivariable logistic regression model of the association between $\geq 4$ comorbidities and the highest quartile of the BRAFMDQ total score. The highest quartile included 48 patients (score range, 18-69) and the lower three quartiles included 142 patients (score range, $0-17$ )

$O R$ odds ratio, $C I$ confidence interval, $C R P$ C-reactive protein, $R A$ rheumatoid arthritis, $R F$ rheumatoid factor, anti-CCP anti-cyclic citrullinated peptide

a Obesity was defined as a body mass index $\geq 30 \mathrm{~kg} / \mathrm{m}^{2}$

Several sensitivity analyses were performed. Use of the untransformed values for CRP in the regression models did not yield different results (Supplementary Table 1). Analysis of the associations between the different numbers of comorbidities and the overall BRAF-MDQ score demonstrated that either $\geq 3$ or $\geq 5$ comorbidities were statistically significantly associated with the overall BRAF-MDQ score (OR 2.35; 95\% CI 1.06-5.27 and OR 8.23; 95\% CI 2.35-33.91, respectively) (Supplementary Table 2). Because subjects who developed RA

Table 4 Adjusted associations between multimorbidity and the BRAF-MDQ total and subscores

\begin{tabular}{lll}
\hline Domain & $\begin{array}{l}\text { Beta coefficient }^{\mathbf{a}} \\
(\mathbf{9 5 \%} \mathbf{C I}) \text { for } \mathbf{2} \mathbf{4} \text { comorbidities }\end{array}$ & $\begin{array}{l}\text { Beta coefficient }^{\mathbf{b}} \\
(\mathbf{9 5 \%} \mathbf{C I}) \text { for number of comorbidities }\end{array}$ \\
\hline Total & $9.33(3.92,14.7)$ & $2.33(1.10,3.56)$ \\
Physical & $2.92(1.18,4.66)$ & $0.76(0.36,1.16)$ \\
Living & $2.90(1.16,4.68)$ & $0.75(0.34,1.15)$ \\
Cognition & $1.86(0.58,3.13)$ & $0.43(0.13,0.72)$ \\
Emotion & $1.79(0.76,2.83)$ & $0.42(0.19,0.66)$ \\
\hline
\end{tabular}

The beta coefficients and 95\% CIs for the associations between multimorbidity and the continuous BRAF-MDQ total score or subdomain scores are shown from multivariable linear regression models. Multimorbidity was defined for each domain as either the presence of $\geq 4$ comorbidities or the continuous (integer) number of comorbidities

$C I$ confidence interval, $R A$ rheumatoid arthritis, $R F$ rheumatoid factor, anti-CCP anti-cyclic citrullinated peptide

a All models are adjusted for age, sex, RA disease duration, obesity, smoking status, RF/anti-CCP positivity, and C-reactive protein

${ }^{\mathrm{b}}$ Coefficient represents the increase in the total BRAF-MDQ corresponding to an increase of 1 comorbidity 
prior to residing in Olmsted County had longer disease duration, separate analyses were performed excluding prevalent cases; no substantive differences were noted (data not shown). Additionally, a separate analysis adjusting for RA medications (i.e., methotrexate, other synthetic DMARDs, biologic DMARDs, and glucocorticoids) was performed; the odds ratio for the association between substantial $(\geq 4)$ multimorbidity and the total BRAF-MDQ score decreased from 3.18 to 2.89 but remained statistically significant $(95 \%$ CI 1.06-7.68, $p=0.035$ ) (Supplementary Table 3).

\section{DISCUSSION}

In this study, we have reported that substantial multimorbidity, defined as the presence of $\geq 4$ comorbidities, is associated with more severe fatigue, after adjusting for important confounders, including age, sex, RA disease duration, obesity, smoking status, RF/anti-CCP positivity, and CRP. Both the number of individual comorbidities and the presence of substantial $(4+)$ multimorbidity were associated with increased fatigue for each of the BRAFMDQ subdomains, including the physical, living, cognition, and emotion domains. However, after further adjustment for the RAPID-3 as a fully patient-reported measure of disease activity, the association between multimorbidity and overall BRAF-MDQ fatigue became statistically non-significant. The RAPID-3 arguably has a high degree of collinearity with the BRAFMDQ because fatigue is a strong determinant of the patient global assessment that is included in the RAPID-3 equation. Therefore, inclusion of RAPID-3 in the model probably represents overadjustment. The magnitude of the adjusted OR (1.76) remains suggestive of a clinically important association between multimorbidity and fatigue even after the RAPID-3 is considered. A future study of larger sample size is necessary to further disentangle the associations between multimorbidity, disease activity, and fatigue in patients with RA.

Prior to this study, relatively little was known about the associations between substantial multimorbidity and the experience of fatigue as reported by patients, particularly on the various subdomains of fatigue as defined by previous qualitative studies. Using data from the multinational QUEST-RA study, Gron et al. reported in 2014 that the number of individual comorbidities was associated with fatigue rated on a $0-10 \mathrm{~cm}$ visual analog scale [35]. In 2018, Tournadre et al. reported in the COMEDRA (COMorbidities EDucation in Rheumatoid Arthritis) study that a multimorbidity index was associated with severe fatigue (numeric rating scale $\geq 5$ of 10) [36]. These previous studies had important limitations. Specifically, Gron et al. did not perform adjustment for disease characteristics other than disease activity and the disability index, and both Gron et al. and Tournadre et al. used simple scales for overall fatigue and, therefore, could not consider the possibility of differential effects of multimorbidity on fatigue subdomains. In the present study, we have provided new information about the magnitude of fatigue as captured using the well validated BRAF-MDQ in the subgroup of patients with more substantial multimorbidity. Furthermore, we have shown that multimorbidity impacts all aspects of fatigue, including the physical, living, cognitive, and emotional subdomains.

How does multimorbidity contribute to fatigue in patients with RA? Central to this question is the potential effect of multimorbidity on RA management and control of disease activity. There is evidence from randomized clinical trials that control of disease activity and attainment of clinical remission is associated with improvement in fatigue [37]. However, the effects of treatment with biologic therapies on RA fatigue are small to moderate, and uncertainty remains about the mechanisms of these improvements, with the possibility of both direct and indirect effects [37, 38]. Additionally, there is evidence, albeit limited, that the presence of multimorbidity is associated with disease activity parameters and may interfere with treatment decision-making by rheumatologists, leading to a lower likelihood of initiating biologic therapies in patients with multimorbidity and contributing to the scenario of 'difficult-to-treat' RA [22, 39, 40]. 
Nonetheless, the preponderance of recent evidence suggests that fatigue is often not mediated by active inflammation in the periphery [6, 12, 41]. Individual comorbidities (e.g., depression, obesity, and pulmonary disease) may contribute directly to fatigue $[1,36]$. Fatigue is tightly linked to pain in patients with RA $[41,42]$, so it is relevant that both pain and fatigue among patients with RA may be mediated by central mechanisms involving altered functional connectivity in the central nervous system [43, 44]. Future studies should explore the impact of multimorbidity on these central mechanisms of fatigue.

Alternatively, the effect of multimorbidity on fatigue could be mediated through treatment complexity and burden on the patient. Qualitative studies have shown that self-management and time management are integral to the experience of fatigue among patients with RA $[9,10,45]$. Studies in the general population have suggested that self-management represents hard work for patients with multimorbidity [46]. The cumulative complexity model posits that patients with multiple chronic medical conditions often have an imbalance between the workload of their health care tasks and the capacity to carry out this work of selfmanagement [47]. The potential imbalance between patients' health care workload and their capacity to manage their disease may contribute importantly to their fatigue experience. Radner et al. have developed a model that is relevant to the potential contribution of healthcare burden to fatigue, whereby patientcentered care for RA should integrate patients' multiple chronic health problems into decisionmaking in order to achieve their treatment goals and enhance their functional status and quality of life [48]. However, further research is necessary to better understand the interactions between multimorbidity, treatment burden, and the fatigue experience for patients with rheumatic diseases.

Strengths of this study include its population-based design, application of a comprehensive multimorbidity index, and use of the BRAFMDQ as a well-validated, patient-centered measure of fatigue. However, several limitations require consideration. The cross-sectional design of this study precluded investigation of the effects of multimorbidity on fatigue over time or with seasonal variation. Composite disease activity measures including joint counts were not performed at the research study visit when patients completed fatigue assessments. However, the RAPID-3 is a validated measure of disease activity and has reasonable correlation with treatment response as defined by the DAS28 [49, 50]. Additionally, it was beyond the scope of this cross-sectional study to understand the interactions between multimorbidity, fatigue, and medications for either RA or other comorbidities. Future longitudinal studies of the relationships between multimorbidity and repeated measures of fatigue over time will provide greater insight into potential causality, especially for patients with DMARD-refractory fatigue. The lack of data on certain comorbidities that are particularly associated with fatigue and reduced physical capacity, such as osteoarthritis, is an important limitation. The study included a primarily white population, limiting generalizability to more diverse groups. Data for socioeconomic status were not available.

\section{CONCLUSIONS}

Multimorbidity is an important contributor to the experience of fatigue among patients with RA, including to the physical, living, cognitive, and emotional aspects of the disease. Physicians should evaluate the impact of multimorbidity when managing fatigue in patients with RA in clinical practice or when judging the comparative effectiveness of disease-modifying therapies for fatigue in RA clinical trials. Future studies are necessary to determine how RA treatment modifies the effects of multimorbidity on fatigue and to explore whether clusters of inter-related comorbidities differentially affect the fatigue experience of patients with RA. The findings suggest that interventions targeting multimorbidity could help alleviate treatmentrefractory fatigue in patients with RA and other rheumatic diseases. 


\section{ACKNOWLEDGEMENTS}

We thank the participants of the study.

Funding. This work was funded by a grant from the National Institutes of Health, NIAMS (R01 AR46849) and made possible by the Rochester Epidemiology Project (R01 AG034676 from the National Institute on Aging). The content is solely the responsibility of the authors and does not necessarily represent the official views of the National Institutes of Health. No Rapid Service Fee was received by the journal for the publication of this article.

Authorship. All named authors meet the International Committee of Medical Journal Editors (ICMJE) criteria for authorship for this article, take responsibility for the integrity of the work as a whole, and have given their approval for this version to be published.

Prior Presentation. The findings of this study have been reported in part at the 2019 American College of Rheumatology annual scientific meeting in Atlanta, Georgia.

Disclosures. John M. Davis III reports a grant from National Institutes of Health, during the conduct of the study, and has received grants from Pfizer and Genentech and served on advisory boards sponsored by AbbVie and Sanofi-Genzyme, outside the submitted work. Cynthia S. Crowson reports a grant from National Institutes of Health, during the conduct of the study, and has received grants from Pfizer and personal fees from Crescendo Biosciences, outside the submitted work. John M. Davis III is a member of the journal's Editorial Board. Elena Myasoedova and Tina M. Gunderson have nothing to disclose.

Compliance with Ethics Guidelines. The Mayo Clinic (IRB number 06-005445) and Olmsted Medical Center (IRB number 039-omc06) institutional review boards approved this research study. The study was conducted in accordance with the Helsinki Declaration of 1964 and its later amendments. All participants provided written informed consent. No protected health information of research participants is disclosed in this manuscript.

Data Availability. The datasets generated during and/or analyzed during the current study are available from the corresponding author on reasonable request.

Open Access. This article is licensed under a Creative Commons Attribution-NonCommercial 4.0 International License, which permits any non-commercial use, sharing, adaptation, distribution and reproduction in any medium or format, as long as you give appropriate credit to the original author(s) and the source, provide a link to the Creative Commons licence, and indicate if changes were made. The images or other third party material in this article are included in the article's Creative Commons licence, unless indicated otherwise in a credit line to the material. If material is not included in the article's Creative Commons licence and your intended use is not permitted by statutory regulation or exceeds the permitted use, you will need to obtain permission directly from the copyright holder. To view a copy of this licence, visit http://creativecommons.org/licenses/by$\mathrm{nc} / 4.0 /$.

\section{REFERENCES}

1. Katz P. Fatigue in rheumatoid arthritis. Curr Rheumatol Rep. 2017;19(5):25.

2. Overman CL, Kool MB, Da Silva JA, Geenen R. The prevalence of severe fatigue in rheumatic diseases: an international study. Clin Rheumatol. 2016;35(2):409-15.

3. Wolfe F, Hawley DJ, Wilson K. The prevalence and meaning of fatigue in rheumatic disease. J Rheumatol. 1996;23(8):1407-17.

4. Odegard S, Kvien TK, Uhlig T. Incidence of clinically important 10-year health status and disease activity levels in population-based cohorts with rheumatoid arthritis. J Rheumatol. 2008;35(1): 54-60.

5. Druce KL, Jones GT, Macfarlane GJ, Verstappen SM, Basu N. The longitudinal course of fatigue in rheumatoid arthritis: results from the Norfolk 
Arthritis Register. J Rheumatol. 2015;42(11): 2059-65.

6. Katz P, Margaretten M, Trupin L, Schmajuk G, Yazdany J, Yelin E. Role of sleep disturbance, depression, obesity, and physical inactivity in fatigue in rheumatoid arthritis. Arthritis Care Res (Hoboken). 2016;68(1):81-90.

7. Nicassio PM, Ormseth SR, Custodio MK, Irwin MR, Olmstead R, Weisman MH. A multidimensional model of fatigue in patients with rheumatoid arthritis. J Rheumatol. 2012;39(9):1807-13.

8. Nikolaus S, Bode C, Taal E, van de Laar MA. Fatigue and factors related to fatigue in rheumatoid arthritis: a systematic review. Arthritis Care Res (Hoboken). 2013;65(7):1128-46.

9. Primdahl J, Hegelund A, Lorenzen AG, Loeppenthin $\mathrm{K}$, Dures E, Appel EB. The experience of people with rheumatoid arthritis living with fatigue: a qualitative metasynthesis. BMJ Open. 2019;9(3):e024338.

10. Hewlett S, Cockshott Z, Byron M, Kitchen K, Tipler $S$, Pope D, et al. Patients' perceptions of fatigue in rheumatoid arthritis: overwhelming, uncontrollable, ignored. Arthritis Rheum. 2005;53(5): 697-702.

11. Ishida M, Kuroiwa Y, Yoshida E, Sato M, Krupa D, Henry N, et al. Residual symptoms and disease burden among patients with rheumatoid arthritis in remission or low disease activity: a systematic literature review. Mod Rheumatol. 2018;28(5): 789-99.

12. Druce KL, Bhattacharya Y, Jones GT, Macfarlane GJ, Basu N. Most patients who reach disease remission following anti-TNF therapy continue to report fatigue: results from the British Society for Rheumatology Biologics Register for Rheumatoid Arthritis. Rheumatology (Oxford). 2016;55(10): 1786-90.

13. Carpenter L, Nikiphorou E, Kiely PDW, Walsh DA, Young A, Norton S. Secular changes in the progression of clinical markers and patient-reported outcomes in early rheumatoid arthritis. Rheumatology (Oxford). 2020;59(9):2381-91.

14. Carpenter L, Barnett R, Mahendran P, Nikiphorou E, Gwinnutt J, Verstappen S, et al. Secular changes in functional disability, pain, fatigue and mental well-being in early rheumatoid arthritis. A longitudinal meta-analysis. Semin Arthritis Rheum. 2020;50(2):209-19.

15. Strand V, Shah R, Atzinger C, Zhou J, Clewell J, Ganguli A, et al. Economic burden of fatigue or morning stiffness among patients with rheumatoid arthritis: a retrospective analysis from real-world data. Curr Med Res Opin. 2020;36:161-8.

16. Nikiphorou E, Nurmohamed MT, Szekanecz Z. Editorial: comorbidity burden in rheumatic diseases. Front Med (Lausanne). 2018;5:197.

17. Radner H. Multimorbidity in rheumatic conditions. Wien Klin Wochenschr. 2016;128(21-22):786-90.

18. Nikiphorou E, Norton S, Carpenter L, Dixey J, Andrew Walsh D, Kiely P, et al. Secular changes in clinical features at presentation of rheumatoid arthritis: increase in comorbidity but improved inflammatory states. Arthritis Care Res (Hoboken). 2017;69(1):21-7.

19. Dougados M, Soubrier M, Antunez A, Balint P, Balsa $\mathrm{A}$, Buch $\mathrm{MH}$, et al. Prevalence of comorbidities in rheumatoid arthritis and evaluation of their monitoring: results of an international, cross-sectional study (COMORA). Ann Rheum Dis. 2014;73(1): $62-8$.

20. Radner H, Smolen JS, Aletaha D. Comorbidity affects all domains of physical function and quality of life in patients with rheumatoid arthritis. Rheumatology (Oxford). 2011;50(2):381-8.

21. Radner H, Smolen JS, Aletaha D. Impact of comorbidity on physical function in patients with rheumatoid arthritis. Ann Rheum Dis. 2010;69(3): 536-41.

22. Radner H, Yoshida K, Frits M, Iannaccone C, Shadick NA, Weinblatt $M$, et al. The impact of multimorbidity status on treatment response in rheumatoid arthritis patients initiating diseasemodifying anti-rheumatic drugs. Rheumatology (Oxford). 2015;54(11):2076-84.

23. Kremers HM, Myasoedova E, Crowson CS, Savova G, Gabriel SE, Matteson EL. The Rochester Epidemiology Project: exploiting the capabilities for population-based research in rheumatic diseases. Rheumatology (Oxford). 2011;50(1):6-15.

24. Arnett FC, Edworthy SM, Bloch DA, McShane DJ, Fries JF, Cooper NS, et al. The American Rheumatism Association 1987 revised criteria for the classification of rheumatoid arthritis. Arthritis Rheum. 1988;31(3):315-24.

25. Pincus $T$, Swearingen CJ, Bergman M, Yazici Y. RAPID3 (Routine Assessment of Patient Index Data 3), a rheumatoid arthritis index without formal joint counts for routine care: proposed severity categories compared to disease activity score and clinical disease activity index categories. J Rheumatol. 2008;35(11):2136-47. 
26. Nicklin J, Cramp F, Kirwan J, Greenwood R, Urban M, Hewlett S. Measuring fatigue in rheumatoid arthritis: a cross-sectional study to evaluate the Bristol Rheumatoid Arthritis Fatigue Multi-Dimensional questionnaire, visual analog scales, and numerical rating scales. Arthritis Care Res (Hoboken). 2010;62(11):1559-68.

27. Dures EK, Hewlett SE, Cramp FA, Greenwood R, Nicklin JK, Urban M, et al. Reliability and sensitivity to change of the Bristol Rheumatoid Arthritis Fatigue scales. Rheumatology (Oxford). 2013;52(10):1832-9.

28. Kirwan J, Coteur G, Dures E, Nicklin J, Bryson J, Hewlett S. Validity and responsiveness of the Bristol Rheumatoid Arthritis Fatigue Multidimensional Questionnaire (BRAF-MDQ) in a randomized controlled clinical trial. Value Health. 2014;17(7): A568-9.

29. Hewlett S, Kirwan J, Bode C, Cramp F, Carmona L, Dures E, et al. The revised Bristol Rheumatoid Arthritis Fatigue measures and the Rheumatoid Arthritis Impact of Disease scale: validation in six countries. Rheumatology (Oxford). 2018;57(2): 300-8.

30. Hewlett S, Almeida C, Ambler N, Blair PS, Choy EH, Dures E, et al. Reducing arthritis fatigue impact: two-year randomised controlled trial of cognitive behavioural approaches by rheumatology teams (RAFT). Ann Rheum Dis. 2019;78(4):465-72.

31. Nicklin J, Cramp F, Kirwan J, Urban M, Hewlett S. Collaboration with patients in the design of patient-reported outcome measures: capturing the experience of fatigue in rheumatoid arthritis. Arthritis Care Res (Hoboken). 2010;62(11):1552-8.

32. Quan H, Sundararajan V, Halfon P, Fong A, Burnand B, Luthi JC, et al. Coding algorithms for defining comorbidities in ICD-9-CM and ICD-10 administrative data. Med Care. 2005;43(11): 1130-9.

33. Moore BJ, White S, Washington R, Coenen $\mathrm{N}$, Elixhauser A. Identifying increased risk of readmission and in-hospital mortality using hospital administrative data: The AHRQ Elixhauser Comorbidity Index. Med Care. 2017;55(7):698-705.

34. England BR, Sayles H, Mikuls TR, Johnson DS, Michaud K. Validation of the rheumatic disease comorbidity index. Arthritis Care Res (Hoboken). 2015;67(6):865-72.

35. Gron KL, Ornbjerg LM, Hetland ML, Aslam F, Khan NA, Jacobs JW, et al. The association of fatigue, comorbidity burden, disease activity, disability and gross domestic product in patients with rheumatoid arthritis. Results from 34 countries participating in the Quest-RA program. Clin Exp Rheumatol. 2014;32(6):869-77.

36. Tournadre A, Pereira B, Gossec L, Soubrier M, Dougados M. Impact of comorbidities on fatigue in rheumatoid arthritis patients: Results from a nurseled program for comorbidities management (COMEDRA). Jt Bone Spine. 2019;86(1):55-60.

37. Almeida C, Choy EH, Hewlett S, Kirwan JR, Cramp $\mathrm{F}$, Chalder T, et al. Biologic interventions for fatigue in rheumatoid arthritis. Cochrane Database Syst Rev. 2016;6:Cd008334.

38. Corominas H, Alegre C, Narvaez J, Fernandez-Cid CM, Torrente-Segarra V, Gomez MR, et al. Correlation of fatigue with other disease related and psychosocial factors in patients with rheumatoid arthritis treated with tocilizumab: ACT-AXIS study. Medicine (Baltimore). 2019;98(26):e15947.

39. Roodenrijs NMT, de Hair MJH, van der Goes MC, Jacobs JWG, Welsing PMJ, van der Heijde D, et al. Characteristics of difficult-to-treat rheumatoid arthritis: results of an international survey. Ann Rheum Dis. 2018;77(12):1705-9.

40. Luque Ramos A, Redeker I, Hoffmann F, Callhoff J, Zink A, Albrecht K. Comorbidities in patients with rheumatoid arthritis and their association with patient-reported outcomes: results of claims data linked to questionnaire survey. J Rheumatol. 2019;46(6):564-71.

41. Druce KL, Jones GT, Macfarlane GJ, Basu N. Determining pathways to improvements in fatigue in rheumatoid arthritis: results from the British Society for Rheumatology Biologics Register for Rheumatoid Arthritis. Arthritis Rheumatol. 2015;67(9):2303-10.

42. van Dartel SA, Repping-Wuts JW, van Hoogmoed D, Bleijenberg G, van Riel PL, Fransen J. Association between fatigue and pain in rheumatoid arthritis: does pain precede fatigue or does fatigue precede pain? Arthritis Care Res (Hoboken). 2013;65(6): 862-9.

43. Basu N, Kaplan CM, Ichesco E, Larkin T, Schrepf A, Murray $\mathrm{AD}$, et al. Functional and structural magnetic resonance imaging correlates of fatigue in patients with rheumatoid arthritis. Rheumatology (Oxford). 2019;58(10):1822-30.

44. Druce KL, Jones GT, Macfarlane GJ, Basu N. Examining changes in central and peripheral pain as mediates of fatigue improvement: results from the British Society for Rheumatology Biologics Register for Rheumatoid Arthritis. Arthritis Care Res (Hoboken). 2016;68(7):922-6. 
45. Feldthusen C, Mannerkorpi K. Factors of importance for reducing fatigue in persons with rheumatoid arthritis: a qualitative interview study. BMJ Open. 2019;9(5):e028719.

46. Rosbach M, Andersen JS. Patient-experienced burden of treatment in patients with multimorbiditya systematic review of qualitative data. PLoS ONE. 2017;12(6):e0179916.

47. Shippee ND, Shah ND, May CR, Mair FS, Montori VM. Cumulative complexity: a functional, patientcentered model of patient complexity can improve research and practice. J Clin Epidemiol. 2012;65(10):1041-51.

48. Radner H, Yoshida K, Smolen JS, Solomon DH. Multimorbidity and rheumatic conditions-enhancing the concept of comorbidity. Nat Rev Rheumatol. 2014;10(4):252-6.
49. Curtis JR, Churchill M, Kivitz A, Samad A, Gauer L, Gervitz L, et al. A randomized trial comparing disease activity measures for the assessment and prediction of response in rheumatoid arthritis patients initiating certolizumab pegol. Arthritis Rheumatol. 2015;67(12):3104-12.

50. Pincus T, Furer V, Keystone E, Yazici Y, Bergman MJ, Luijtens K. RAPID3 (Routine Assessment of Patient Index Data 3) severity categories and response criteria: Similar results to DAS28 (Disease Activity Score) and CDAI (Clinical Disease Activity Index) in the RAPID 1 (Rheumatoid Arthritis Prevention of Structural Damage) clinical trial of certolizumab pegol. Arthritis Care Res (Hoboken). 2011;63(8):1142-9. 